University of Wollongong

Research Online

Faculty of Informatics - Papers (Archive)

Faculty of Engineering and Information

Sciences

$12-12-2006$

\title{
Fringe calibration using neural network signal mapping for structured light
} profilometers

\author{
Matthew J. Baker \\ matthewb@uow.edu.au \\ Jiangtao Xi \\ University of Wollongong, jiangtao@uow.edu.au \\ Joe F. Chicharo \\ University of Wollongong, chicharo@uow.edu.au
}

Follow this and additional works at: https://ro.uow.edu.au/infopapers

Part of the Physical Sciences and Mathematics Commons

\section{Recommended Citation}

Baker, Matthew J.; Xi, Jiangtao; and Chicharo, Joe F.: Fringe calibration using neural network signal mapping for structured light profilometers 2006.

https://ro.uow.edu.au/infopapers/532

Research Online is the open access institutional repository for the University of Wollongong. For further information contact the UOW Library: research-pubs@uow.edu.au 


\title{
Fringe calibration using neural network signal mapping for structured light profilometers
}

\author{
Abstract \\ We present a novel neural network signal calibration technique to improve the performance of \\ triangulation based structured light profilometers. The performance of such profilometers is often \\ hindered by the capture of noisy and aberrated pattern intensity distributions. We address this problem by \\ employing neural networks and a spatial digital filter in a signal mapping approach. The performance of \\ the calibration technique is gauged through both simulation and experimentation, with simulation results \\ indicating that accuracy can be improved by more than $80 \%$.

\section{Disciplines} \\ Physical Sciences and Mathematics

\section{Publication Details} \\ This paper was originally published as: Baker, MJ, Xi, J \& Chicharo, JF, Fringe calibration using neural \\ network signal mapping for structured light profilometers, International Symposium on Intelligent Signal \\ Processing and Communications 2006 (ISPACS '06), Yonago, Japan, 12-15 December 2006, 784-787. \\ Copyright IEEE 2006.
}




\title{
Fringe Calibration using Neural Network Signal Mapping for Structured Light Profilometers
}

\author{
Matthew J. Baker* Jiangtao Xi* and Joe F. Chicharo* \\ ${ }^{*}$ School of Electrical, Computer and Telecommunications Engineering, \\ University of Wollongong, NSW 2522, Australia \\ Tel: +6124221 3244, Fax: +61242273277 \\ E-mail:mjb06@uow.edu.au
}

\begin{abstract}
We present a novel neural network signal calibration technique to improve the performance of triangulation based structured light profilometers. The performance of such profilometers is often hindered by the capture of noisy and aberrated pattern intensity distributions. We address this problem by employing neural networks and a spatial digital filter in a signal mapping approach. The performance of the calibration technique is gauged through both simulation and experimentation, with simulation results indicating that accuracy can be improved by more than $80 \%$.
\end{abstract}

\section{INTRODUCTION}

Structured light techniques for non-contact, dynamic and accurate profile measurement of diffuse surfaces have been widely studied due to their potential industrial applications. The most exploited techniques often utilise a projected periodic fringe pattern composed of parallel lines. The observed pattern is distorted by the diffuse surface in such a way that represents information about the height of the object perpendicular to the plane of observation. The distorted structured light pattern is recorded, commonly by a CCD camera and through computer analysis of the recorded image the object can be recreated in 3D space, typically with a high degree of precision. Figure 1 depicts the typical Crossed Optical Axes geometry utilised by many traditional structured light profilometers. Using geometrical relationships apparent in the arrangement the physical height distribution of the object can be extracted. Noting that $\triangle E_{p} E_{c} D$ and $\triangle A C D$ are similar it can be shown that.

$$
\begin{gathered}
\frac{A C}{-h(x, y)}=\frac{d_{0}}{l_{0}-h(x, y)} \\
h(x, y)=\frac{l_{0} A C}{A C-d_{0}},
\end{gathered}
$$

Thus, assuming that system parameters $d_{0}$ and $l_{0}$ are known, the aim of any profilometry algorithm is to accurately determine spatial distance $A C$.

Commonly, $A C$ is processed as a spatial phase value using popular Fourier Transform [1] or Phase Measuring Profilometry [2] based techniques. These techniques require the projection and capture of a sinusoidal intensity distribution. When the sinusoidal intensity distribution is projected onto the diffuse surface of interest the distribution can be regarded as a phase modulated version of the initial reference signal, with

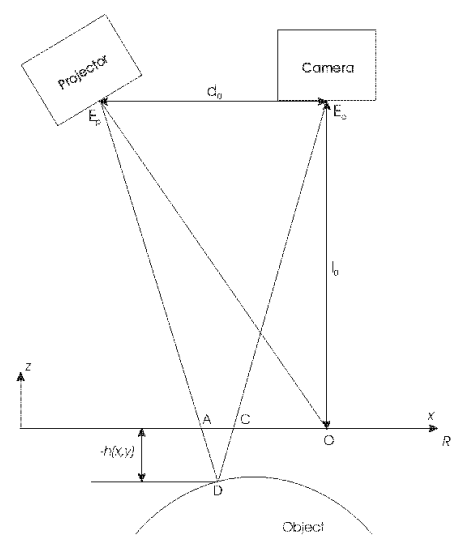

Fig. 1. Typical Crossed Optical Axes profilometry arrangement

the modulation directly related to the depth distribution of the diffuse surface. Often such structured light techniques are nonresilient to nonlinear aberrations and other noise present in captured fringe patterns and as a result the ability to accurately determine $A C$ is significantly hindered. These fringe anomalies typically result from nonlinear camera / projector intensity responses, fringe image preprocessing prior to digital projection and camera / projector lens distortion.

Some interesting solutions utilise neural networks to directly detect $A C$ from fringe data [3] or in calibration applications to enhance the ability for reconstruction algorithms to detect $A C$ [4], [5], [6]. In this paper we describe a neural network calibration approach whereby an aberrated noise corrupt captured intensity distribution is spatially filtered then input to a feed-forward backpropagation neural network trained using non-aberrated patterns. We exploit the generalisation properties of neural networks to interpolate modulated structured light patterns and consequentially are successfully capable of improving the accuracy in which $A C$ can be determined and hence reduce surface profile reconstruction errors. The method only requires a single image for calibration and thus is suitable for applications such as robotic vision where rapid calibration is required. Such an approach is desirable as it is not limited to operation with particular pattern structures and can successfully function independent of any reconstruction 


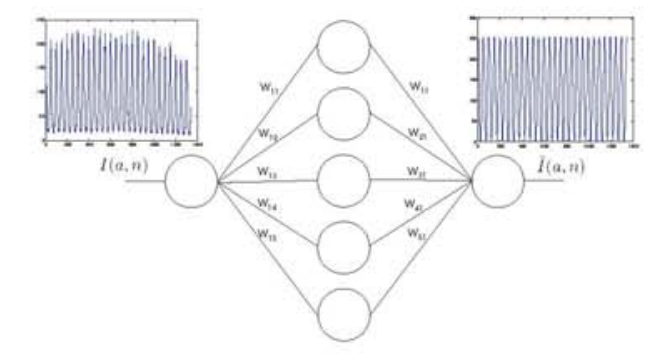

Fig. 2. Proposed Multilayer Signal Mapping Calibration Neural Network for arbitrary $a \in M$

algorithm

\section{Proposed Calibration Technique}

\section{A. Principle Neural Network Technique}

The neural network based calibration technique endeavors to reproduce captured structured light patterns devoid of aberration from captured aberrated data via a multilayer feedforward backpropagation neural network operating as a nonlinear signal map. The calibration technique considers $I$, a $M \times N$ digitised aberrated image of a fringe map projected onto a reference plane. $M$ neural networks such as that depicted in Figure 2 are trained to function as a non-linear signal map such that when the aberrated image $I$ is then applied to the neural networks, $\hat{I}$, a calibrated version of the initial image is produced. Hence, mathematically:

$$
\begin{array}{r}
\hat{I}(m, n)=f_{2}\left(\sum W_{m_{2}} f_{1}\left(I(m, n) W_{m_{1}}\right)\right) \\
\text { for } n=0,1,2,3 \ldots \ldots . N, \\
\text { and } m=0,1,2,3 \ldots . M,
\end{array}
$$

where $\hat{I}(m, n)$ is the output of the $m t h$ neural network for the $n t h$ sample corresponding to the $m t h$ and $n t h$ sample of the calibrated reference image, $W_{m_{1}}$ and $W_{m_{2}}$ are the corresponding weight matrices and $f_{1}()$ and $f_{2}()$ are the appropriate activation functions for the corresponding layers. The $M$ neural networks are then used to obtain calibrated fringe images in which are then input to a structured light reconstruction algorithm such as PMP or FTP.

Whilst neural networks can be applied to solve many problems over a range of different disciplines due to their nonlinear mapping abilities, in the presence of random processes such as additive gaussian noise the performance of neural networks for such purposes can be somewhat limited, hence, an adequate noise removal technique is required to maximize the performance of the neural calibration.

\section{B. Noise Removal}

Traditionally, the removal of noise from a fringe image will involve some form of filtering, typically, the filtering will be either performed in the spatial frequency domain [7] via fft and multiplying operations or in the spatial domain [8], [9], [10] through convolution, adaptive or non-linear processing. Since in spatial frequency techniques it is required to calculate the Fourier and Inverse Fourier Transform of the signal of interest, for real-time processing systems such as dynamic structured light profilometers, we are more incline to employ convolutive spatial domain techniques. Spatial domain techniques utilise neighborhood spatial coherence and neighborhood pixel value homogeneity as a basis. Spatial filters replace incoherent pixel values with values more spatially coherent by using surrounding neighboring pixels [11].

Gaussian smoothing is a noise suppression technique where a "bell-shaped" gaussian kernel is utilised to act as a spatial lowpass filter in which blurs an image. A typical 1-D gaussian kernel is formed as:

$$
G(x)=k e^{-\frac{x^{2}}{2 \sigma^{2}}}
$$

for all $x \in W$, where,

$$
k=\frac{1}{\sum_{x \in W} e^{-\frac{x^{2}}{2 \sigma^{2}}}}
$$

and $W$ is the width of the gaussian kernel. If we consider the gaussian operator seen in equation (3) in the spatial frequency domain it too is of similar gaussian form. In contrast to a mean or averaging filter with a rectangular kernel which oscillates in the spatial frequency domain, we can be confident which spatial frequencies will be attenuated based on the selected gaussian parameters [12].

Since the phase modulation an object introduces into a fringe pattern is considered to be slowly varying in contrast to the fringe carrier frequency and more importantly the signal noise, the blurring caused by the Gaussian smoothing process introduces minimal error provided the appropriate kernel parameters are selected.

It is important to note that in this case we have chosen a filtering technique based on the particular characteristics of the noise present in the fringe pattern. Obviously a gaussian smoothing technique will perform poorly in the presence of "salt and pepper" or impulsive noise, due to its point spreading nature. A more appropriate spatial filter for such an application would be the non-linear median filter [12] a technique Ryoo and Choi implemented in their analysis of noisy white-light interferograms [10].

\section{Simulation}

To validate and also gauge the performance of the proposed calibration technique we simulate the reconstruction of a diffuse surface illuminated by a nonlinear aberrated / noise affected fringe sequence. We model a reference sinusoidal with a $20 \mathrm{~dB}$ second order harmonic component corresponding to a typical captured digitally projected sinusoidal distribution:

$$
\begin{array}{r}
r_{n}(x, y)=128+100 \cos \left(2 \pi f_{0} x+2 \pi n / N\right) \\
+10 \cos \left(2 \pi\left(2 f_{0}\right) x+4 \pi n / N\right), \\
\text { for } n=0,1,2 \ldots . N-1
\end{array}
$$




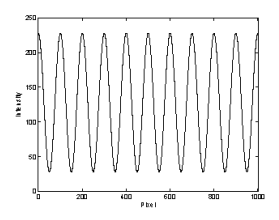

(a) Reference Signal

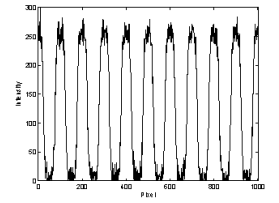

(c) Noisy Signal $\overline{r_{n}}(x, y)$

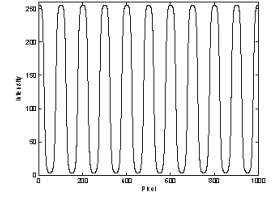

(b) Distorted Signal $\xi\left(r_{n}(x)\right)$

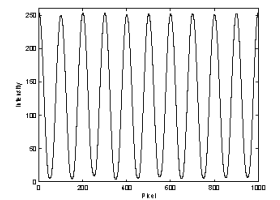

(d)
$\hat{r_{n}} f(x, y)$

Fig. 3. Simulated Signal Cross-sections for $n=0$

Consequentially, we define our deformed phase modulated fringe as

$$
\begin{array}{r}
d_{n}(x, y)=128+100 \cos \left(2 \pi f_{0} x+\phi(x, y)+2 \pi n / N\right) \\
+10 \cos \left(2 \pi\left(2 f_{0}\right) x+4 \pi n / N+2 \phi(x, y)\right) \\
\text { for } n=0,1,2 \ldots N-1
\end{array}
$$

where $\phi(x, y)$ represents a hemispherical convex shape as seen in Figure 4(a). The camera / projector nonlinear intensity response is modelled as:

$$
\xi(s)=128 \frac{\tanh \left(\frac{3 s}{128}-3\right)}{\tanh (3)}+128,
$$

Subsequent to these processes the signals are corrupt with additive gaussian noise yielding a common Signal to Noise Ratio of $20 \mathrm{~dB}$. Thus, our distorted noise corrupt reference fringe sequence $\overline{r_{n}}(x, y)$ and distorted noise corrupt deformed phase modulated fringe sequence $\bar{d}_{n}(x, y)$ are given as

$$
r_{n}^{-}(x, y)=\xi\left(r_{n}(x)\right)+n_{n}^{r}(x)
$$

and

$$
\bar{d}_{n}(x, y)=\xi\left(d_{n}(x)\right)+n_{n}^{d}(x)
$$

respectively, where $n_{n}^{r}(x)$ and $n_{n}^{d}(x)$ are the noise vectors introduced into into the $n t h$ reference and phase modulated signals respectively. Figure 3(a) - (c) displays a cross-section of the the initial reference sinusoidal signal, the nonlinear distorted signal and the noise corrupt signal respectively. The distorted noise corrupt signals $\overline{r_{n}}(x, y)$ and $\overline{d_{n}}(x, y)$ were filtered using the gaussian smoothing technique described in Subsection II.B to give

$$
{\overline{r_{n}}}^{f}(x, y)=\sum_{k}^{m} \overline{r_{n}}(x+k-1, y) \cdot G(k),
$$

\begin{tabular}{c|cc|} 
Input Fringe & $\epsilon_{e}\left(\mathrm{rads}^{-1}\right)$ & $\sigma_{e}\left(\mathrm{rads}^{-1}\right)$ \\
\hline Non-Calibrated & 0.0992 & 0.0763 \\
Calibrated & 0.0965 & 0.0755 \\
Calibrated-Filtered & 0.0152 & 0.0118
\end{tabular}

TABLE I

MEan ABsolute Phase ERror and Standard Deviation, PMP

$$
N=3
$$

and

$$
\begin{array}{r}
{\overline{d_{n}}}^{f}(x, y)==\sum_{k}^{m} \overline{d_{n}}(x+k-1, y) \cdot G(k), \\
\text { for } x=1,2 \ldots M-m+1
\end{array}
$$

where $m$ is the width of the gaussian kernel $G()$ and $M$ is the width of the fringe distribution in samples. Through trial and error, $m$ and $\sigma$ of the gaussian kernel was determined to be 35 and 13.5 respectively. Figure 3(d) displays a cross-section of the filtered noise corrupt signal.

Since the fringe non-linear anomalies are independent of $y$ we trained a single neural network as described in Subsection II.A employing a single hidden layer of 5 neurons utilising a tansigmoidal activation function $f$ and a pure linear output stage. Each set of $n$ signals, $\overline{r_{n}}(x, y), \overline{d_{n}}(x, y)$ and the filtered versions $\overline{r_{n}} f(x, y),{\overline{d_{n}}}^{f}(x, y)$ for both the reference and deformed signals were applied to the trained neural network to yield the calibrated fringe patterns $\hat{r_{n}}(x, y), \hat{d_{n}}(x, y)$ and ${\hat{r_{n}}}^{f}(x, y){\hat{d_{n}}}^{f}(x, y)$.

A standard 3 step PMP technique $(N=3)$ was used to extract the phase distribution $\phi(x, y)$ for the filtered calibrated, the non-filtered calibrated and also the non-calibrated fringe sequences. Table 1 displays the mean absolute phase reconstruction error $\epsilon_{e}$ along with corresponding standard deviation $\sigma_{e}$ for both the calibrated and non-calibrated situations. For the calibrated-filtered case the absolute mean measurement error has been improved from $0.0992 \mathrm{rads}^{-1}$ to 0.0152 $\mathrm{rads}^{-1}$ improving the accuracy by more than $84 \%$. Clearly the performance of the neural network is significantly hindered with the introduction of noise with only a less than $3 \%$ improvement on accuracy under the specified noise conditions without filtering. Figure 4 displays the complete reconstruction of the simulated hemispherical diffuse surface for filtered calibrated, non-filtered calibrated and non-calibrated fringe sequences. It is clearly evident visually the improvement in reconstruction for filtered calibrated case in contrast to the non-filtered calibrated and non-calibrated cases. We will now consider the experimental application of the proposed neural network technique.

\section{EXPERIMENTATION}

In order to verify the physical application of the noise removal and calibration technique, practical experimental results were established through the profiling of a diffuse object. A simusoidal fringe pattern was projected using an InFocus LP530 digital video projector and captured using a MS3100 


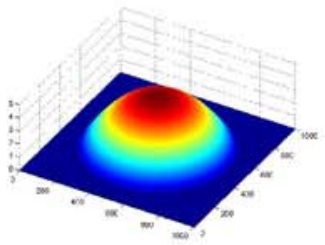

(a) Simulated Phase Distribution $\phi(x, y)$

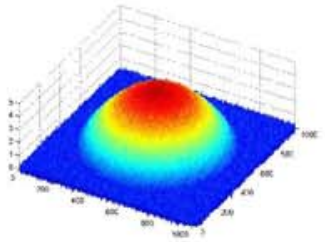

(c) Calibrated Non-filtered Reconstruction

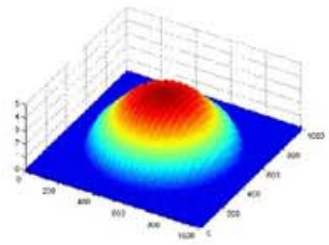

(b) Non-calibrated Reconstruction

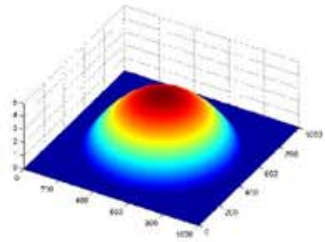

(d) Filtered Calibrated Reconstruction
Fig. 4. Simulated Reconstructed Phase Distributions

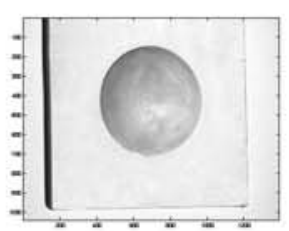

(a) Experimental surface

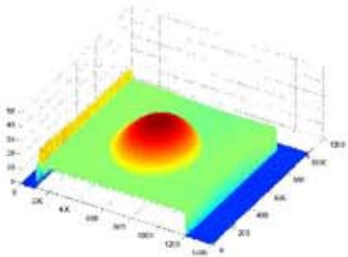

(c) Non-filtered Calibrated

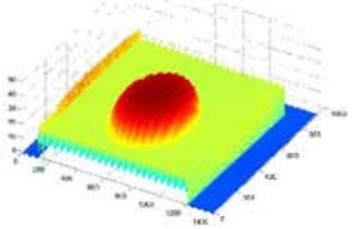

(b) Non-calibrated Reconstruction

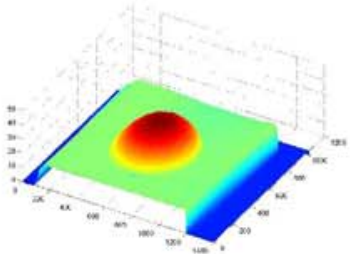

(d) Filtered Calibrated
Fig. 5. Experimental object and fringe patterns

3-CCD camera. The resolution of the CCD camera is 1392 x 1039 pixels with a field of vision $260 \times 194 \mathrm{~mm}^{2}$. This corresponds to a captured fringe pattern with a spatial resolution of $0.1868 \mathrm{~mm} /$ pixel, yielding a spatial period of $25.7 \mathrm{~mm}$ equating to a spatial frequency $f_{0}$ of 38.9 fringes $/ \mathrm{m}$. System parameters $l_{0}$ and $d_{0}$ were measured to be $2 \mathrm{~m}$ and $0.81 \mathrm{~m}$ respectively. The profiled surface was a convex dome shape as seen in Figure 5(a). The distorted fringe image was calibrated and filtered using the neural network approach describe in Section III, with the guassian kernel parameters $m$ and $\sigma$ determined as 40 and 13.5 respectively. The maximum height of the hemispherical surface is $22.8 \mathrm{~mm}$ with a diameter of $99 \mathrm{~mm}$ with the thickness of the base material being $16 \mathrm{~mm}$. Figures 5(b)-(d) display the reconstructed surfaces for nonfiltered / non-calibrated, non-filtered / calibrated and filtered / calibrated cases. The performance of the calibration technique

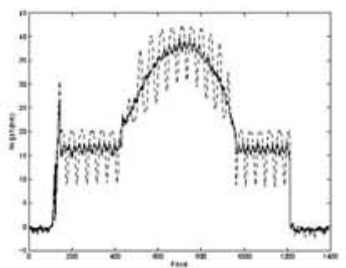

(a) Calibrated Non-filtered Reconstruction

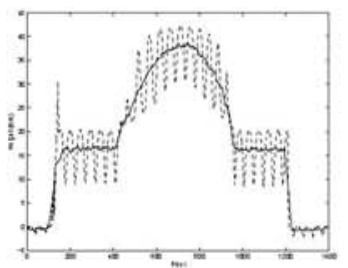

(b) Filtered Calibrated Reconstruction
Fig. 6. Cross-section of reconstructed diffuse surface seen in Figure 5(a)

is clearly evident with the filtered and calibrated providing the most accurate reconstruction of the diffuse surface. Figures 6(a) and (b) further emphasize the improvement displaying a cross-section of the reconstructed dome surface. The dashed line indicating the non-filtered / non-calibrated case and the solid lines indicating the non-filtered and filtered for Figures 6(a) and (b) respectively.

\section{CONCLUSION}

In this paper we have proposed a neural network signal mapping calibration technique, aimed at reducing noise and nonlinear aberrations present in captured structured light patterns. We have verified the application through simulation and also empirically, and have shown significantly improved surface profile reconstruction results. The method only requires a single image for calibration and thus is suitable for dynamic applications where haste calibration is required.

\section{REFERENCES}

[1] X. Su, W. Chen, "Fourier transform profilometry: a review," Optics and Lasers in Engineering, vol. 35, pp. 263-284, 2001.

[2] V. Srinivasan, H. C. Lui, M. Halioua, "Automated phase-measuring profilometry of 3-d diffuse objects," Applied Optics, vol. 23, pp. 3105$3108,1984$.

[3] F. J. Cuevas, M. Servin, O. N. Stavroudis, R. Rodriguez-Vera, "Multilayer neural network applied to phase and depth recovery from fringe patterns," Optics Communications, vol. 181, pp. 239-259, July 2000.

[4] F. J. Cuevas, M. Servin, R. Rodriguez-Vera, "Depth object recovery using radial basis functions," Optics Communications, vol. 163, pp. 270 277, May 1999.

[5] M. Chang W. Tai, "360-deg profile noncontact measurement using a neural network," Optical Engineering, vol. 34, no. 12, pp. 3572-3576, December 1995.

[6] G. Zhang, Z. Wei, "A novel calibration approach to structured light 3d vision inspection," Optics and Laser Technology, vol. 34, pp. 373-380, 2002.

[7] J. Xu, Y. Wang, S. Si, C. Gao, D. Yun, "Research on application of special filter in projecting grating profilometry," Optical Technology and Image Processing for Fluids and Solids Diagnostics SPIE, vol. 5058, pp. $532-536,2003$

[8] J. Villa, M.Servin, L. Castillo, "Profilometry for the measurement of 3-d object shapes based on regularized filters," Optics Communications, vol. 161, pp. 13-18, January 1999.

[9] H. Zhi, R. B. Johansson, "Adaptive filter for enhancement of fringe patterns," Optics and Lasers in Engineering, vol. 15, pp. 241-251, 1991

[10] S. Ryoo, T. Choi, "3-d profilometry by analysis of noisy white-light interferograms," In three-dimensional and Multidimensional Microscopy: Image Acquisition Processing VII SPIE, vol. 3919, pp. 152-160, 2000.

[11] R. M. Haralick, L. G. Shapiro, Computer and Robot Vision. AddisonWesley, 1992, vol. 1.

[12] E. R. Davies, Machine Vision: Theory Algorithms Practicalities, 3rd ed. Elsevier, 2005. 